\title{
Efficient anti-collision algorithm using variable length ID in RFID systems
}

\author{
Woon-Young Yeo ${ }^{1}$ and Gyung-Ho Hwang ${ }^{2 \mathrm{a})}$ \\ ${ }^{1}$ Department of Information and Communications Engineering, Sejong University, \\ 98 Gunja-dong, Gwangjin-gu, Seoul, 143-747 Korea \\ ${ }^{2}$ Division of Information Communication and Computer Engineering, \\ Hanbat National University, San 16-1, Dukmyong-Dong, Yuseong-gu, Daejeon, \\ 305-719, Korea \\ a)gabriel@hanbat.ac.kr
}

\begin{abstract}
The RFID tags are used for low cost and convenient object identification. For the fast tag identification, the anti-collision algorithm is used. A new algorithm to identify RFID tags using a shortened ID instead of a long original tag ID is proposed. The proposed method changes the length of the shortened ID adaptively according to the number of unidentified tags. The proposed algorithm shows better performance compared to previous anti-collision algorithms in terms of average transmitted bits per one tag identification.
\end{abstract}

Keywords: RFID, anti-collision, query tree

Classification: Wireless circuits and devices

\section{References}

[1] D. K. Klair, K.-W. Chin, and R. Raad, "A Survey and Tutotial of RFID Anti-Collision Protocols," IEEE Commun. Survey and Tutorials., April 2010.

[2] K. W. Chiang, C. Hua, and T. P. Yum, "Prefix-Randomized Query-Tree Protocol for RFID Systems," Proc. IEEE Int. Conf. Commun., pp. 16531657, June 2006.

[3] K. W. Chiang, C. Hua, and T. P. Yum, "Prefix-Length Adaptation for PRQT Protocol in RFID Systems," Proc. IEEE Global Telecommunications Conference, pp. 1-5, Nov. 2006.

[4] G. Binetti, G. Boggia, P. Camarda, and L. A. Grieco, "A Hashing-based Anti-Collision Algorithm for RFID Tag Identification," Proc. Int. Symposium on Wireeless Communication Systems, ISWCS 2007, pp. 524-528, Oct. 2007.

[5] J.-B. Eom and T.-J. Lee, "Accurate Tag Estimation for Dynamic FramedSlotted ALOHA in RFID Systems," IEEE Commun. Lett., vol. 14, no. 1, pp. 60-62, Jan. 2010.

[6] EPC Global, "EPC radio-frequency protocols class-1 generation-2 UHF RFID protocol for communications at $860 \mathrm{MHz}-960 \mathrm{MHz}$ version 1.2.0," Oct. 2008.

[7] J. H. Choi, D. Lee, and H. Lee, "Query Tree-based Reservation for Efficient RFID Tag Anti-Collision," IEEE Commun. Lett., vol. 11, no. 1, pp. 85-87, Jan. 2007. 


\section{Introduction}

Radio Frequency Identification (RFID) systems are widely used due to its ability to identify objects by wireless even in the non line-of-sight. RFID systems consist of RF tags and readers. The tags are able to communicate with readers through a shared radio channel. The readers collects the identifiers (IDs) of tags.

There are passive and active tags. The passive tags get a power from reader's transmitted radio and respond at reader commands. The active tags have long radio coverage with battery powered circuits. The passive tags are more popular because of their low cost and simple circuits. In the reader's neighborhood, it is possible that the passive tags can transmit their IDs to a reader at the same time. The collisions prevent the reader to obtain the tag IDs, which makes the tag identification time to be longer. The one of main issues in RFID systems is an anti-collision protocol which is critical for the performance. In this paper, we propose a new efficient anti-collision algorithm using a shortened ID instead of original long ID for the identification.

\section{RFID Anti-collision algorithms}

Anti-collision algorithms are divided into two methods, deterministic method and stochastic method according to different approaches [1].

The deterministic methods are based on tree protocols. The basic idea of tree protocols is to divide tags into sub-trees and identifies only one tag at last. There are several methods such as query tree, binary searching, tree splitting and bitwise arbitration. In this paper, we focus on the query tree based protocols where the reader sends a query and when the tag's prefix is equal to that of the query, the tags respond to a reader with their IDs. The query tree algorithm has lowest tag cost and system cost compared to other methods since the tag need to have prefix matching and synchronization circuits. The query tree algorithm is an efficient RFID tag identification method which guarantees that all the passive tags are read.

The stochastic methods are based on aloha protocols. The reader tells each tag to choose a time slot and the tags transmit their own IDs in a randomly selected time slot. If two or more tags select the same time slot, a collision occurs and the tags should retransmit their ID during next query procedure. The dynamic framed slotted aloha (DFSA) algorithm has best performance in case that the frame size is equal to the number of tags. The tag number estimation is one issue of DFSA scheme and one of the methods is proposed in [5].

There are some previous researches on the query tree algorithm to improve the performance. In [2], each tag generates a $k$ bits random binary prefix and the reader polls each of the $2^{k}$ prefixes sequentially. When the tags are polled with the same prefix, the tag sends its original ID. When the collision occurs since two more tags have the same prefix, the tags generate 1 bit as ' 0 ' or ' 1 ' and the reader polls the 1 bit longer prefix again. This polling is not an efficient method because all the $2^{k}$ prefixes need to be checked. Moreover, 
after a random prefix is identified, the original ID should be transmitted, which is also waste of resource. They also proposed an initial prefix length adaptation algorithm in [3]. In the case that total size of tags is unknown, the algorithm sets the initial prefix length as small $l$ length and polls all the $2^{l}$ prefix and find the optimal initial prefix size that satisfies the collision ratio. However, the method to find optimal initial prefix size is a time-consuming procedure because all the cases should be polled and the collision ratio needs to be calculated. The authors in [4] proposed a hybrid anti-collision algorithm where a short ID that is the output of hash function of original entire ID is used for the query tree algorithm and if the hash results are collided, the aloha based protocol is used. However the optimal ID length is obtained by simulations and the results are obtained using a fixed ID length. In [7], the authors proposed a query tree-based reservation algorithm in which the tags use 16 bits random number instead of original long ID. The complete ID is sent after the tag is identified by the reader. Even though the 16 bits are long enough to exclude the collision due to the same random number ID, the fixed size random ID is still inefficient and the method could be improved for a better performance.

\section{Proposed efficient anti-collision algorithm using variable length ID}

The proposed anti-collision algorithm is based on query tree algorithm in which the tags use variable length ID instead of an entire long ID. We call the shortened ID to be used for identifications as SID.

In EPG global standard, the RFID tag has 96 bits length known as the General Identifier (GID-96) including header ( 8 bits), general manager number (28 bits), object class (24 bits) and serial number (36 bits) [6]. As shown in Fig. 1, the SID has the right part of the original ID and the length of a SID is variable. Because the serial number field has 36 bits length that is long enough to be a SID, it can be inferred that the SID has good properties for randomness. In the proposed algorithm, the right most bit of a SID is treated as the first bit in the SID. That is, a SID size is increased as reverse direction unlike the original ID. Using this scheme, the variable length SID can be adapted to the query tree algorithm.

The Fig. 2 shows the procedure of the proposed algorithm. The initial

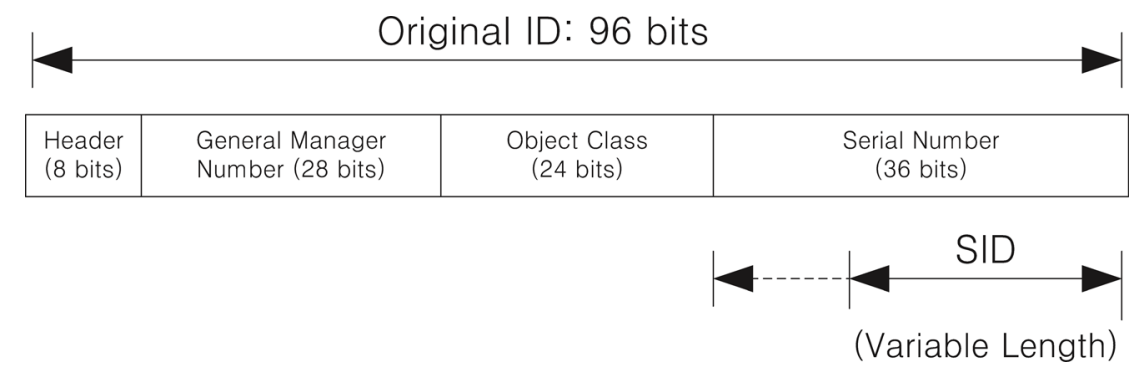

Fig. 1. EPG GID-96 and the proposed Shortened ID (SID) 


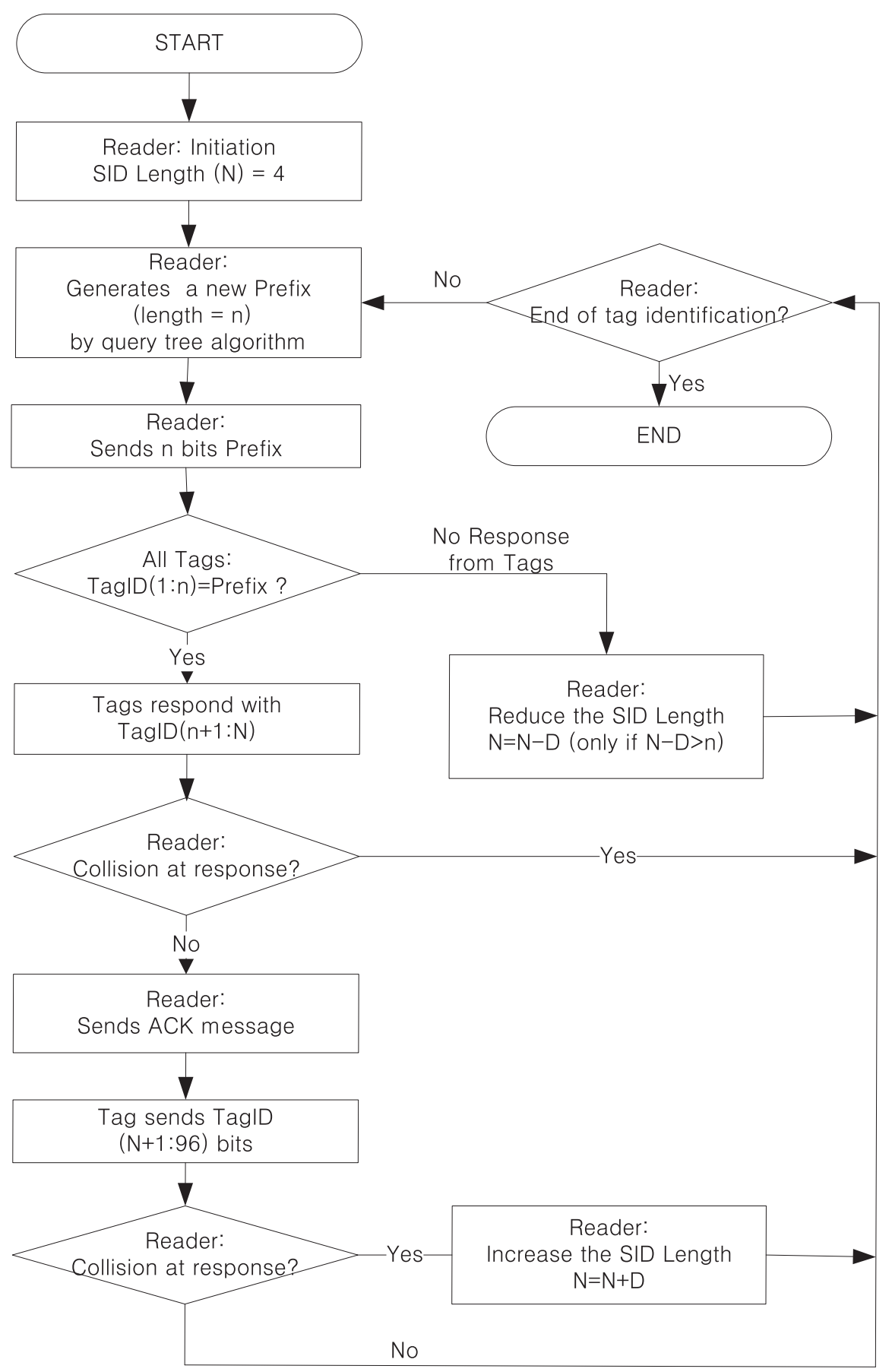

Fig. 2. Efficient anti-collision algorithm using variable length ID

length of SID is set to 4 bits which are proper for the small number of tags. Let $N$ be the SID length in bits. The information on SID length $(N)$ is included in a query frame that is transmitted by a reader. The reader queries with a prefix of $n$ bits generated by the query tree algorithm where the first prefix is ' 0 ' and 1 bits. If the prefix is equal to the tag's SID, the tag sends the remaining part of SID that has $N-n$ bits length. In case that there's collision at tags responses, the reader transmits a query with a newly generated prefix based on the rules of query tree algorithm. If the tag's response is identified with ACK message by the reader, the tag sends its ID except the SID part. 
If there are more than two tags to have same SID, the transmitted remaining bits of an original ID are collided. In this case, the length of SID is extended by the predefined size, $D$ bits and new prefix is sent by the reader. On the contrary, the length of SID is decreased by $D$ bits when there's no response at the reader's prefix query which means that the number of unidentified tags may be small. The reduction of the SID length occurs only when the equation $N-D>n$ is satisfied because the new SID length should be larger than the current prefix. Since the proposed algorithm can change the length of SID adaptively according to the number of unidentified tags the algorithm can reduce the total transmitted bits for identifications.

In the case that a fixed size random ID is used for anti-collision algorithm, the original long ID should be transmitted after the random ID is identified. But the proposed algorithm uses a shortened SID extracted from the original ID and when a tag is identified, only the part of the original ID except the SID is transmitted.

\section{Performance evaluation and results}

We assume that the wireless channel is error-free between a single reader and multiple tags. The number of tags changes from 10 to 500 . The original tag ID has 96 bits length. For the performance comparisons, we used the query tree algorithm where the Manchester coding is not used. Thus, the exact positions of the collided bits when two more tags send their bits at the same time are not notified.

The simulation results are shown in Fig. 3. The average transmitted bits per one tag identification of the proposed scheme are much lower than those of the query tree algorithm. In the case that the 16 bits random ID is used, the transmitted bits are more than those of the proposed method because the 16 bits is long for small number of tags and the entire original ID need to be transmitted after the 16 bits random ID is identified.

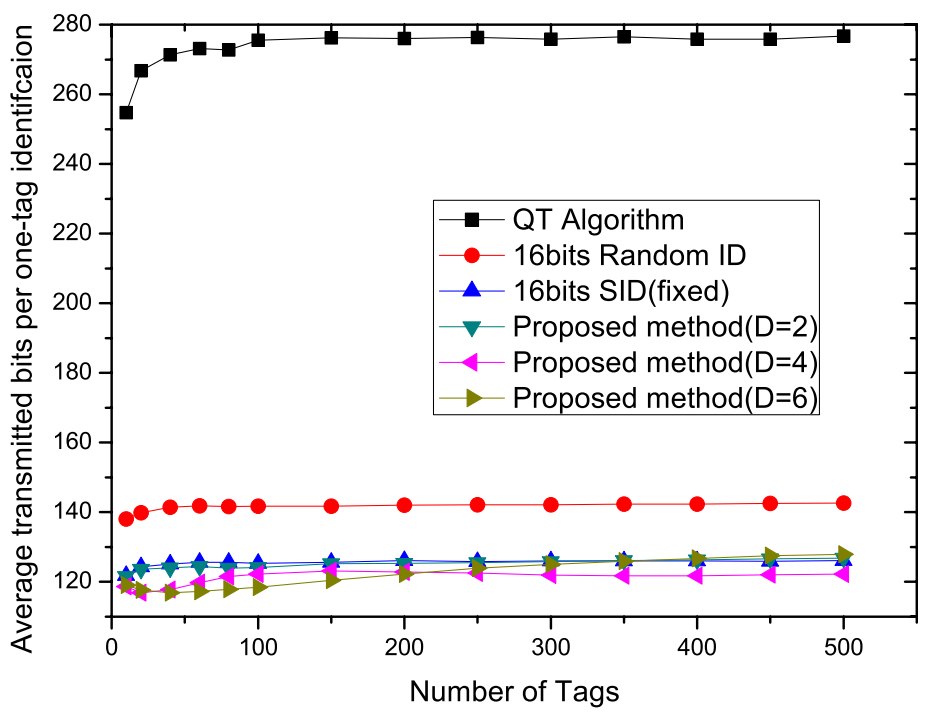

Fig. 3. The average transmitted bits per one tag identification 
The query tree scheme using 16 bits fixed size SID is also evaluated and the performance of that method is worse than that of the proposed method using the variable length SID. The fixed size SID cannot be optimal in the case that the number of unidentified tags and the collision probability of the SIDs are changed. Since the proposed method uses the variable length SID for the identification, the required bits for one tag identification is smaller than the methods which use the fixed size shortened IDs. And according to the predefined adjustment size of a SID, that is $D$, the performance is slightly varied and the performance with adjustment size of $D=4$ has best performance overall.

\section{Conclusions}

The new anti-collision algorithm using variable length ID in RFID systems has been proposed. The proposed method has a better performance in the terms of required transmission bits for one-tag identification compared to the original query tree algorithm because the shortened ID for identification has a variable length based on the collisions and idle responses. The simulation result shows that the proposed method has a better performance than query tree algorithm using a fixed length random ID for identifications.

\section{Acknowledgments}

This research was supported by the MKE (The Ministry of Knowledge Economy), Korea, under the national HRD support program for convergence information technology supervised by the NIPA (National IT Industry Promotion Agency) (NIPA-2010-C6150-1001-0013). 